\title{
A Model for Fine Mapping in Family Based Association Studies
}

\author{
Stefan Boehringer ${ }^{a}$ Ruth M. Pfeiffer ${ }^{b}$

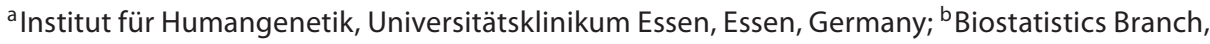 \\ National Cancer Institute, Bethesda, Md., USA
}

\section{Key Words}

Family data • Likelihood • Haplotypes • Latent disease locus • Alzheimer

\begin{abstract}
Genome wide association studies for complex diseases are typically followed by more focused characterization of the identified genetic region. We propose a latent class model to evaluate a candidate region with several measured markers using observations on families. The main goal is to estimate linkage disequilibrium (LD) between the observed markers and the putative true but unobserved disease locus in the region. Based on this model, we estimate the joint distribution of alleles at the observed markers and the unobserved true disease locus, and a penetrance parameter measuring the impact of the disease allele on disease risk. A family specific random effect allows for varying baseline disease prevalences for different families. We present a likelihood framework for our model and assess its properties in simulations. We apply the model to an Alzheimer data set and confirm previous findings in the ApoE region.
\end{abstract}

Copyright $\odot 2009$ S. Karger AG, Basel

\section{Introduction}

Recently, single nucleotide polymorphism (SNP) based genome wide association studies (GWASs) using unrelated cases (individuals with disease) and controls (indi- viduals without disease), have proven to be a feasible approach to identify SNPs associated with complex disease phenotypes [1-5]. However, once a genomic region is identified, follow up studies are needed to better understand the biology underlying the disease process.

Fine mapping regions identified by GWASs and identifying disease associated SNPs or haplotypes can be done using two basic designs: case-control or family studies. While case-control association studies using unrelated cases and controls are more powerful [6], family based association studies are robust to population stratification that might lead to false positive findings $[7,8]$. In addition, ascertaining families with multiple affected members might reduce genetic heterogeneity by enriching alleles with bigger effect sizes $[9,10]$.

We propose a likelihood based approach to analyzing family data that incorporates segregation information about an unobserved 'true' disease locus hypothesized to be located in the same region as the observed marker loci. We assume that the penetrance function for a binary phenotype (presence or absence of a disease) depends only on this true latent disease locus and a family specific random effect. We model the joint distribution of alleles at the observed loci and alleles at the unobserved disease associated locus in the region. In addition to haplotype frequencies at the observed loci, this joint distribution is characterized by the allele frequencies at the latent disease locus and linkage disequilibrium (LD) between the latent disease locus and observed loci, thus directly guiding the exploration of associated genomic regions. It can be esti-

\section{KARGER}

Fax +4161306 1234 E-Mail karger@karger.ch www.karger.com
(C) 2009 S. Karger AG, Basel

0001-5652/09/0674-0226\$26.00/0

Accessible online at:

www.karger.com/hhe
Stefan Boehringer

Institut für Humangenetik, Universitätsklinikum Essen

Hufelandstr. 55, DE-45122 Essen (Germany)

Tel. +49 201723 4533, Fax +49 2017355900

E-Mail correspondence@s-boehringer.org 
mated using data on family members. Assuming that the disease prevalence in the population is known, we also estimate the penetrance parameter for the true disease locus in a logistic model for disease. We describe our approach for families that were randomly sampled from the population, as well as for families that were ascertained on the basis of single or multiple affected members.

Models based on a latent true disease locus have been proposed previously [11-13]. However, [13] assumed that individuals sampled into the study were unrelated, [11] focused on testing rather than estimation, and [12] used an estimating equation rather than a likelihood based approach. A more detailed discussion of this related work is presented in the last section.

The rest of the paper is structured as follows: the data and probability model and the corresponding likelihood and are given in section 2 . In section 3 we use simulations to investigate properties of our model. We apply the methods to an Alzheimer data set in section 4 and close with a discussion in section 5 .

\section{Data and Likelihood}

\section{Data}

We assume that $N$ nuclear families are sampled into our study, with $n_{i}$ denoting the family size of family $i$. The phenotypes of the $i$-th family are $\mathbf{Y}_{\mathrm{i}}=\left(Y_{1}, Y_{2}, Y_{i 1}, \ldots, Y_{i n_{\mathrm{i}}}\right)$. For families that consist of two parents and offspring, indices 1 and 2 designate parents and indices $3, \ldots, n_{i}$ denote offspring. The phenotype is a binary trait, that is $Y_{i j}=1$ for affected and $Y_{i j}=0$ for unaffected individuals.

We assume the true disease locus is unobserved, but $K$ marker loci in the same region as the disease locus are measured for each individual. Such a region would typically span a range of 50-100 $\mathrm{kb}$, depending on the population and the specific location in the genome [14]. We denote the observed multi-locus genotype of individual $j$ in the $i$-th family by $G_{i j}=\left(G_{i j 1}, \ldots, G_{i j K}\right)$. The observed joint genotypes for the $i$-th nuclear family are $\mathbf{G}_{\mathbf{i}}=\left(G_{i 1}, \ldots, G_{i n_{i}}\right)$.

The observed data for each family thus consist of phenotypes and genotypes at the observed loci, $\left(\mathbf{Y}_{\mathbf{i}}, \mathbf{G}_{\mathbf{i}}\right)=\left(Y_{i 1}, \ldots, Y_{i n_{\mathbf{i}}}\right.$, $\left.G_{i 1}, \ldots, G_{i n_{\mathrm{i}}}\right)$.

\section{The Penetrance Model}

The probability of disease of individual $j$ in the $i$-th family depends on the unobserved genotype $G_{i j}^{*}$ at the latent disease locus, with alleles $a$ and $A$. We assume that $A$ is the disease associated allele with allele frequency $p^{*}=P(A)$. The following scores model the genotype for an assumed mode of inheritance: $X_{i j}^{*}=0$ for $G_{i j}^{*}=\{a a\}, X_{i j}^{*}=1$ for $G_{i j}^{*}=\{A A\}$, and $X_{i j}^{*}=k$ for $G_{i j}^{*}=\{a A\}$, where $k=0$ for a recessive mode of inheritance, $k=1$ for a dominant mode of inheritance, and $k=1 / 2$ for an additive mode of inheritance.

The penetrance function is given by

$$
\operatorname{logit}\left(p_{i j}\right)=\operatorname{logit}\left\{P\left(Y_{i j}=1 \mid X_{i j}^{*}, z_{i}\right)\right\}=\mu+\sigma_{z} z_{i}+\beta X_{i j}^{*} .
$$

Here, $\mu$ is the common intercept, that we assume is known from external data. The family specific random intercept $z_{i}$ allows for different baseline penetrances for different families, and follows a normal distribution with mean 0 and variance 1 . For identifiability purposes, we assume that $\beta \neq 0$. Implications of this assumption are discussed later. The above penetrance function can be extended to include other measured covariates $U_{i j}$ using $\operatorname{logit}\left\{P\left(Y_{i j}=1 \mid X_{i j}^{*}, U_{i j}\right)\right\}=\mu+\sigma_{z} z_{i}+\beta X_{i j}^{*}+\gamma U_{i j}$.

Under the logistic model (1), the marginal probability of the response in the $i$-th family requires integration over the random effects distribution, an operation that cannot be carried out in closed form, and is written as

$P\left(\mathbf{Y}_{\mathbf{i}} \mid \mathbf{X}_{\mathbf{i}}^{*}\right)=P\left(Y_{i 1}, \ldots, X_{i n_{i}}^{*} \mid X_{i 1}^{*}, \ldots, X_{i n_{i}}^{*}\right)=\int \prod_{j=1}^{n_{i}} p_{i j}^{y_{i j}} q_{i j}^{1-y_{i j}} d F(z)$,

where $q_{i j}=1-p_{i j}$. We also note that $G^{*}$ is the only genotype influencing disease risk in the region, thus leading to conditional independence of phenotypes and the observed genotypes:

$$
\begin{aligned}
& P\left(\mathbf{Y}_{\mathbf{i}} \mid \mathbf{G}_{\mathbf{i}}, \mathbf{G}_{\mathbf{i}}^{*}\right)=P\left(\mathbf{Y}_{\mathbf{i}} \mid \mathbf{G}_{\mathbf{i}}^{*}\right)=P\left(\mathbf{Y}_{\mathbf{i}} \mid \mathbf{X}_{\mathbf{i}}^{*}\right)= \\
& P\left(Y_{i 1}, \ldots, Y_{i n_{\mathrm{i}}} \mid X_{i 1}^{*}, \ldots, X_{i n_{\mathbf{i}}}^{*}\right)
\end{aligned}
$$

When $\sigma_{z}^{2}=0$, model (1) reduces to standard logistic regression and $P\left(\mathbf{Y}_{\mathbf{i}} \mid \mathbf{X}_{\mathbf{i}}^{*}\right)=\prod_{j=1}^{n_{\mathrm{i}}} P\left(Y_{i j} \mid X_{i j}^{*}\right)$.

\section{Likelihood for a Candidate Region}

First, we assume that families in our study are a random sample of families in the population. While our model can be applied to various family structures, for ease of exposition we assume each family consists of two parents and $n_{i}-2$ offspring.

The pair of haplotypes for individual $j$ in family $i$ for the $K$ observed loci is denoted by $H_{i j}=\left(H_{i j}^{1}, H_{i j}^{2}\right)$. Haplotypes formed by observed loci and the unobserved disease locus are denoted by $H_{i j}^{*}=\left(H_{i j}^{* 1}, H_{i j}^{* 2}\right)$. In the general population, haplotypes are assumed to arise from a multinomial distribution with probabilities $P\left(H_{i j}=h_{k}\right)=\eta_{k}$ for $k=1, \ldots, 2^{K}$ and $P\left(H_{i j}^{*}=h_{k}^{*}\right)=\eta_{k}^{*}$ for $k=1, \ldots, 2^{K+1}$, respectively. Under the assumption of Hardy-Weinberg equilibrium (HWE), $P\left(H_{i j}=\left(h_{1}, h_{2}\right)\right)=\left(\eta_{h_{1}}\right)^{2}$ if $h_{1}=h_{2}$ and $2 \eta_{h_{1}} \eta_{h_{2}}$ otherwise with analogous expressions for $P\left(H_{i j}^{*}\right)$.

Using (3), Mendelian inheritance, the assumption of no recombinations in the observed region, and the fact that the genetic contributions of offspring are independent, conditional on the parental pair of haplotypes, the likelihood for $N$ families is obtained by summation over the true unobserved disease genotype:

$$
\begin{aligned}
L(\theta) & =P(\mathbf{Y}, \mathbf{G}, \theta)=\prod_{i=1}^{N} P\left(\mathbf{Y}_{\mathbf{i}}, \mathbf{G}_{\mathbf{i}}\right)=\prod_{i=1}^{N} \sum_{\mathbf{g}_{\mathbf{i}}^{*}} P\left(\mathbf{Y}_{\mathbf{i}}, \mathbf{G}_{\mathbf{i}}, \mathbf{G}_{\mathbf{i}}^{*}=\mathbf{g}^{*}\right) \\
= & \prod_{i=1}^{N} \sum_{\mathbf{g}_{i}=\left(\mathbf{h}_{\mathbf{i}}^{*} \mid \mathbf{h}_{\mathbf{i}}^{* 2}\right)} P\left(\mathbf{Y}_{\mathbf{i}}, \mathbf{H}_{\mathbf{i}}^{*}=\mathbf{h}_{\mathbf{i}}^{*}\right) \\
= & \prod_{i=1}^{N} \sum_{\mathbf{g}_{i}=\left(\mathbf{h}_{\mathbf{i}}^{* 1} \mid \mathbf{h}_{\mathbf{i}}^{* 2}\right)} P\left(\mathbf{Y}_{\mathbf{i}} \mid \mathbf{G}_{\mathbf{i}}^{*}=\mathbf{g}_{\mathbf{i}}^{*}\right) P\left(H_{i 3}^{*}=h_{i 3}^{*}, \ldots, H_{i n_{i}}^{*}=h_{i n_{i}}^{*} \mid h_{i 1}^{*}, h_{i 2}^{*}\right) \\
& \times P\left(H_{i 1}^{*}=h_{i 1}^{*}\right) P\left(H_{i 2}^{*}=h_{i 2}^{*}\right) \\
= & \prod_{i=1}^{N} \sum_{\mathbf{g}_{i}=\left(\mathbf{h}_{\mathbf{i}}^{* 1} \mid \mathbf{h}_{\mathbf{i}}^{* 2}\right)} P\left(\mathbf{Y}_{\mathbf{i}} \mid \mathbf{g}_{\mathbf{i}}^{*}\right) \prod_{j=3}^{n_{i}} P\left(H_{i j}^{*}=h_{i j}^{*} \mid h_{i 1}^{*}, h_{i 2}^{*}\right) \prod_{j=1}^{2} P\left(H_{i j}^{*}=h_{i j}^{*}\right)
\end{aligned}
$$


Here, $\mathbf{g}_{i}=\left(\mathbf{h}_{\mathbf{i}}^{* 1} \mid \mathbf{h}_{\mathbf{i}}^{* 2}\right)$ denotes the set of haplotype combinations of the $i$-th family compatible with the observed genotypes $\mathbf{g}_{\mathbf{i}}$.

The penetrance function $P\left(\mathbf{Y}_{\mathbf{i}} \mid \mathbf{G}_{\mathbf{i}}^{*}\right)$ depends on parameters $\beta$ and $\sigma_{z}$ as defined in (1). Given the parental pair of haplotypes, the genetic contributions of offspring $P\left(H_{i j}^{*} \mid h_{i 1}^{*}, h_{i 2}^{*}\right)$ follow from Mendelian inheritance and thus do not need to be parameterized. There are several parameterizations that can be used for the parental genetic information $P\left(\mathbf{H}^{*}\right)$, all depending on $2^{K+1}-1$ parameters, where $K$ denotes the number of observed loci.

One of the goals of this work is to estimate LD between the disease allele and haplotypes at the observed loci. For two biallelic loci with minor alleles $A$ and $B$, with allele frequencies $p_{A}$ and $p_{B}$ and haplotype frequency $p_{A B}$ of $\mathrm{AB}, \delta$ is defined as $\delta=p_{A B}-p_{A} p_{B}$ [15]. Recall that $p^{*}$ denotes the allele frequency of the disease associated allele $A$ at the latent disease locus. The joint distribution of $H^{*}$ is characterized by LD between haplotypes $H$ corresponding to the observed genotypes and the disease allele $A$, i.e. $\delta_{h}=$ $p_{h A}-p_{h} p_{A}$ for haplotype $h$. The $\delta$ s satisfy the constraint $\Sigma_{i=1}^{\left(2^{K}\right)} \delta_{i}=$ 0 . One parameterization of the likelihood (4) is therefore given by $\theta=\theta_{1}=\left(\eta_{1}, \ldots, \eta_{2^{K}-1}, \delta_{1}, \ldots, \delta_{2^{K}-1}, p^{*}, \beta, \sigma_{z}\right)$.

Alternatively to $\delta$, we use the correlation [15] between haplotype $h$ and the disease allele,

$$
R_{i}=\frac{\delta_{i}}{\sqrt{p^{*}\left(1-p^{*}\right)} \sqrt{\eta_{i}\left(1-\eta_{i}\right)}}
$$

and parameterize (4) using $\theta=\theta_{2}=\left(\eta_{1}, \ldots, \delta_{2^{K}{ }_{-1}}, R_{1}, \ldots, R_{2^{K}-1}, p^{*}\right.$, $\left.\beta, \sigma_{z}\right)$.

\section{Inference}

Identifiability of the likelihood based on the logistic penetrance function without the random effect is shown in [16]. The maximum likelihood estimates (MLEs) $\hat{\theta}$ are found by direct maximization of the likelihood function (4).

\section{Asymptotic Theory}

According to standard theory, the MLEs are asymptotically normally distributed:

$$
\sqrt{n}(\hat{\theta}-\theta) \rightarrow N\left(0, I^{-1}(\theta)\right),
$$

with

$$
(I(\theta))_{j k}=-E\left[\frac{\partial^{2}}{\partial \theta_{j} \partial \theta_{k}} \ln L(\mathbf{Y}, \mathbf{G} ; \theta)\right] .
$$

Recall, that for identifiability purposes we assume $\beta \neq 0$. The null hypothesis of interest for our model thus is that there is no disease locus linked to the observed loci, i.e. $H_{0}: \delta_{1}=\ldots=\delta_{2^{K}-1}=$ 0 or equivalently $H_{0}: R_{1}=\ldots=R_{2^{K}-1}=0$, which can be evaluated using a likelihood ratio test:

$$
T=2\left(\sup _{\theta \in \Theta_{1}} l(\theta \mid Y, \mathrm{G})-\sup _{\theta \in \Theta_{0}} l(\theta \mid Y, \mathrm{G})\right) \sim \chi_{2^{K}-1}^{2},
$$

where $\Theta_{1}=\left(\eta_{1}, \ldots, \eta_{2^{K}-1}, \delta_{1}, \ldots, \delta_{2^{K}}-1, p^{*}, \beta, \sigma_{z}\right)$ and $\Theta_{0}=\left(\eta_{1}, \ldots\right.$, $\left.\eta_{2^{K}-1}, 0, \ldots, 0, p^{*}, \beta, \sigma_{z}\right)$ is the restriction of $\Theta_{1}$ on $\delta_{i}=0$ for all $i$.

\section{Implementation}

We implemented the maximization using the software package $\mathrm{R}$ (http://r-project.org) with the function optim(BFGS algo- rithm). Numerical integration was performed with a Romberg algorithm. To be able to use unconstrained optimization, we parameterized allele and haplotype frequencies using a polytomous logistic function, $\eta_{i}=e^{\gamma_{i}} /\left(1+\Sigma_{j} e^{\gamma_{i}}\right)$. For LD parameters $R$ that fall into $(-1,1)$ we used the parameterization $R=-1+2\left(1+e^{-\lambda}\right)^{-1}$ and $\sigma_{z}$ was parameterized as $\sigma_{z}=\exp (\zeta)$. Additional constraints on the LD parameters given by the marginal haplotype frequencies and $p^{*}$ were checked for every evaluation of the likelihood. The Fisher information matrix was computed using numerical differentiation. Source code of the implementation is available upon request from the first author.

Direct optimization allows for a simultaneous analysis of approximately three SNPs for moderate sample sizes ( $\sim 400$ families). For five or more observed loci, the high dimensionality of the parameter space prevents the use of direct optimization. As an alternative, a sliding window approach can be used for analysis of a larger number of SNPs, by moving a window of two, three or four neighboring SNPs across a given region.

\section{Ascertainment Correction}

For rare diseases, random samples of families generally do not yield enough cases for robust inference. To enrich the sample with affected individuals, families included in a study are thus typically required to have one or more affected family members. For proper inference, the likelihood needs to be conditioned on the ascertainment event. For example, if families are ascertained based on one or more affected family members, the corresponding likelihood is:

$$
\begin{aligned}
& L(\theta)=\prod_{i=1}^{N} P\left(Y_{i 1}, \ldots, Y_{i n_{i}}, G_{i 1}, \ldots, G_{i n_{i}} \mid \sum_{j=1}^{n_{i}} Y_{i j} \geq 1\right) \\
& =\prod_{i=1}^{N} \frac{P\left(Y_{i 1}, \ldots, Y_{i n_{i}}, G_{i 1}, \ldots, G_{i n_{i}}\right)}{P\left(\sum_{j=1}^{n_{i}} Y_{i j} \geq 1\right)} \\
& =\prod_{i=1}^{N} \frac{P\left(Y_{i 1}, \ldots, Y_{i n_{i}}, G_{i 1}, \ldots, G_{i n_{i}}\right)}{1-P\left(\sum_{j=1}^{n_{i}} Y_{i j}=0\right)} .
\end{aligned}
$$

We also study this ascertainment scheme in the simulations below.

\section{Simulations}

We assess the performance of our model on simulated data. To generate observations on families, we use the following steps. Haplotypes $H^{*}$ that included the true disease locus are drawn independently from a multinomial distribution for the two parents. Haplotypes of offspring are generated from parental diplotypes using Mendelian inheritance. For each family, a random effect $z_{i}$ is drawn from a $N(0,1)$ distribution. Given $\mathbf{G}^{*}$ and $z_{i}$, the phenotypes $Y_{i j}$ are drawn from a binomial distribution with probability $p_{i j}$, given by (1). For the analysis, $G^{*}$, phase 
Table 1. Mean estimates over 100 or 500 (for misspecification) simulations for randomly sampled families

\begin{tabular}{|c|c|c|c|c|c|c|}
\hline FS & $\mathrm{N}$ & $\eta_{1}=0.25$ & $R_{1}=0.00$ & $p^{*}=0.25$ & $\beta=1.00$ & $\sigma_{z}=1.00$ \\
\hline \multicolumn{7}{|c|}{ Simulation: additive; estimation: additive } \\
\hline FS1 & 400 & $0.25(0.00)$ & $-0.00(0.01)$ & $0.25(0.00)$ & $1.04(0.18)$ & $0.99(0.16)$ \\
\hline FS2 & 400 & $0.25(0.00)$ & $-0.00(0.01)$ & $0.25(0.00)$ & $0.66(0.24)$ & $0.50(0.21)$ \\
\hline FS3 & 400 & $0.25(0.00)$ & $0.00(0.01)$ & $0.25(0.00)$ & $0.64(0.26)$ & $0.53(0.22)$ \\
\hline FS4 & 300 & $0.25(0.00)$ & $-0.00(0.00)$ & $0.25(0.00)$ & $1.04(0.18)$ & $0.98(0.14)$ \\
\hline FS5 & 300 & $0.25(0.00)$ & $0.00(0.01)$ & $0.25(0.00)$ & $0.76(0.22)$ & $0.69(0.12)$ \\
\hline FS6 & 300 & $0.25(0.00)$ & $-0.00(0.01)$ & $0.25(0.00)$ & $0.73(0.20)$ & $0.71(0.14)$ \\
\hline \multicolumn{7}{|c|}{ Simulation: dominant; estimation: dominant } \\
\hline FS1 & 400 & $0.25(0.00)$ & $0.00(0.01)$ & $0.25(0.00)$ & $1.03(0.14)$ & $0.98(0.15)$ \\
\hline FS2 & 400 & $0.25(0.00)$ & $-0.00(0.01)$ & $0.25(0.00)$ & $0.65(0.14)$ & $0.55(0.16)$ \\
\hline FS3 & 400 & $0.25(0.00)$ & $0.00(0.01)$ & $0.25(0.00)$ & $0.65(0.15)$ & $0.59(0.17)$ \\
\hline FS4 & 300 & $0.25(0.00)$ & $0.00(0.01)$ & $0.25(0.00)$ & $1.03(0.18)$ & $0.99(0.16)$ \\
\hline FS5 & 300 & $0.25(0.00)$ & $-0.00(0.01)$ & $0.25(0.00)$ & $0.70(0.19)$ & $0.73(0.15)$ \\
\hline FS6 & 300 & $0.25(0.00)$ & $-0.00(0.01)$ & $0.25(0.00)$ & $0.68(0.16)$ & $0.75(0.12)$ \\
\hline FS & $\mathrm{N}$ & $\eta_{1}=0.25$ & $R_{1}^{2}=0.50$ & $p^{*}=0.25$ & $\beta=1.00$ & $\sigma_{z}=1.00$ \\
\hline \multicolumn{7}{|c|}{ Simulation: additive; estimation: additive } \\
\hline FS1 & 400 & $0.25(0.00)$ & $0.50(0.00)$ & $0.25(0.00)$ & $1.01(0.16)$ & $0.98(0.14)$ \\
\hline FS2 & 400 & $0.25(0.00)$ & $0.50(0.00)$ & $0.25(0.00)$ & $0.65(0.22)$ & $0.47(0.24)$ \\
\hline FS3 & 400 & $0.25(0.00)$ & $0.50(0.00)$ & $0.25(0.00)$ & $0.68(0.23)$ & $0.42(0.24)$ \\
\hline FS4 & 300 & $0.25(0.00)$ & $0.50(0.00)$ & $0.25(0.00)$ & $1.04(0.16)$ & $1.00(0.13)$ \\
\hline FS5 & 300 & $0.25(0.00)$ & $0.50(0.00)$ & $0.25(0.00)$ & $0.75(0.20)$ & $0.69(0.16)$ \\
\hline FS6 & 300 & $0.25(0.00)$ & $0.50(0.00)$ & $0.25(0.00)$ & $0.78(0.19)$ & $0.70(0.13)$ \\
\hline \multicolumn{7}{|c|}{ Simulation: dominant; estimation: dominant } \\
\hline FS1 & 400 & $0.25(0.00)$ & $0.50(0.00)$ & $0.25(0.00)$ & $1.00(0.14)$ & $0.98(0.15)$ \\
\hline FS2 & 400 & $0.25(0.00)$ & $0.50(0.00)$ & $0.25(0.00)$ & $0.67(0.13)$ & $0.53(0.15)$ \\
\hline FS3 & 400 & $0.25(0.00)$ & $0.50(0.00)$ & $0.25(0.00)$ & $0.68(0.12)$ & $0.52(0.15)$ \\
\hline FS4 & 300 & $0.25(0.00)$ & $0.50(0.00)$ & $0.25(0.00)$ & $0.99(0.15)$ & $1.00(0.15)$ \\
\hline FS5 & 300 & $0.25(0.00)$ & $0.50(0.00)$ & $0.25(0.00)$ & $0.73(0.14)$ & $0.72(0.14)$ \\
\hline FS6 & 300 & $0.25(0.00)$ & $0.50(0.00)$ & $0.25(0.00)$ & $0.75(0.13)$ & $0.73(0.13)$ \\
\hline
\end{tabular}

A total of $\mathrm{n}=1200$ individuals were simulated. Standard deviations are given in parentheses. FS denotes family structure (families contain either both parents and 1 (FS1) or 2 offspring (FS4), one parent and 2 (FS2) or 3 offspring (FS5) or are comprised only of 3 (FS3) or 4 siblings (FS6)).

$\mathrm{N}=$ number of families; $\mu=-2.00$.

information and - for some family structures - parents are removed from the data set.

For each set of simulations all families have the same size and structure. To study the effect of family structure on estimates, we simulated families with both parents and 1 (FS1) or 2 offspring (FS4), one parent and 2 (FS2) or 3 offspring (FS5) and families comprised only of 3 (FS3) or 4 siblings (FS6). The respective study sizes were chosen so that the overall number of individuals, and thus genotyping cost, was constant. We also studied our model on samples of families ascertained on the basis of having at least one affected offspring, since this was the ascertainment for the families in our data example. For ascertained family simulations, we added one offspring to each family structure (FS1a, ..., FS6a).

\section{Parameter Estimates}

Table 1 shows mean parameter estimates over 100 simulated data sets for randomly sampled families. The data for this table were created assuming no LD between the true disease locus and one observed marker locus in the first section and varying $\mathrm{LD}$ otherwise. The allele frequencies of both, the observed locus and the unobserved locus were estimated very accurately; $\hat{\eta}_{1}=0.25$ for all settings with standard errors less than 0.005 , and $\hat{p}^{*}=0.25$, also with standard errors below 0.005 . Similarly, esti- 
Table 2. Mean estimates over 100 simulations for randomly sampled families

\begin{tabular}{|c|c|c|c|c|c|c|c|c|c|c|}
\hline FS & $\mathrm{N}$ & $\eta_{1}=0.10$ & $\eta_{2}=0.16$ & $\eta_{3}=0.37$ & $R_{1}=0.24$ & $R_{2}=0.13$ & $R_{3}=-0.12$ & $p^{*}=0.20$ & $\beta=1.00$ & $\sigma_{z}=1.00$ \\
\hline \multicolumn{11}{|c|}{ Simulation: additive; estimation: additive } \\
\hline FS1 & 400 & $0.10(0.00)$ & $0.16(0.00)$ & $0.37(0.00)$ & $0.24(0.00)$ & $0.13(0.00)$ & $-0.12(0.00)$ & $0.20(0.00)$ & $1.01(0.02)$ & $1.01(0.02)$ \\
\hline FS2 & 400 & $0.10(0.00)$ & $0.16(0.00)$ & $0.37(0.00)$ & $0.24(0.00)$ & $0.13(0.00)$ & $-0.13(0.00)$ & $0.20(0.00)$ & $0.66(0.22)$ & $0.41(0.40)$ \\
\hline FS3 & 400 & $0.10(0.00)$ & $0.16(0.00)$ & $0.37(0.00)$ & $0.24(0.00)$ & $0.13(0.00)$ & $-0.13(0.00)$ & $0.20(0.00)$ & $0.65(0.22)$ & $0.44(0.38)$ \\
\hline FS4 & 300 & $0.10(0.00)$ & $0.16(0.00)$ & $0.37(0.00)$ & $0.24(0.00)$ & $0.13(0.00)$ & $-0.13(0.00)$ & $0.20(0.00)$ & $1.04(0.05)$ & $0.98(0.02)$ \\
\hline FS5 & 300 & $0.10(0.00)$ & $0.16(0.00)$ & $0.37(0.00)$ & $0.24(0.00)$ & $0.13(0.00)$ & $-0.12(0.00)$ & $0.20(0.00)$ & $0.72(0.20)$ & $0.69(0.12)$ \\
\hline FS6 & 300 & $0.10(0.00)$ & $0.16(0.00)$ & $0.37(0.00)$ & $0.24(0.00)$ & $0.13(0.00)$ & $-0.12(0.00)$ & $0.20(0.00)$ & $0.71(0.21)$ & $0.67(0.14)$ \\
\hline \multicolumn{11}{|c|}{ Simulation: dominant; estimation: dominant } \\
\hline FS1 & 400 & $0.10(0.00)$ & $0.16(0.00)$ & $0.37(0.00)$ & $0.24(0.00)$ & $0.13(0.00)$ & $-0.12(0.00)$ & $0.20(0.00)$ & $1.02(0.06)$ & $1.00(0.04)$ \\
\hline FS2 & 400 & $0.10(0.00)$ & $0.16(0.00)$ & $0.37(0.00)$ & $0.24(0.00)$ & $0.13(0.00)$ & $-0.12(0.00)$ & $0.20(0.00)$ & $0.60(0.21)$ & $0.55(0.24)$ \\
\hline FS3 & 400 & $0.10(0.00)$ & $0.16(0.00)$ & $0.37(0.00)$ & $0.24(0.00)$ & $0.13(0.00)$ & $-0.12(0.00)$ & $0.20(0.00)$ & $0.58(0.23)$ & $0.58(0.21)$ \\
\hline FS4 & 300 & $0.10(0.00)$ & $0.16(0.00)$ & $0.37(0.00)$ & $0.24(0.00)$ & $0.13(0.00)$ & $-0.12(0.00)$ & $0.20(0.00)$ & $0.96(0.08)$ & $1.00(0.04)$ \\
\hline FS5 & 300 & $0.10(0.00)$ & $0.16(0.00)$ & $0.37(0.00)$ & $0.24(0.00)$ & $0.13(0.00)$ & $-0.13(0.00)$ & $0.20(0.00)$ & $0.64(0.21)$ & $0.74(0.10)$ \\
\hline FS6 & 300 & $0.10(0.00)$ & $0.16(0.00)$ & $0.37(0.00)$ & $0.24(0.00)$ & $0.13(0.00)$ & $-0.13(0.00)$ & $0.20(0.00)$ & $0.61(0.23)$ & $0.73(0.10)$ \\
\hline \multicolumn{11}{|c|}{ Simulation: recessive; estimation: recessive } \\
\hline FS1 & 400 & $0.10(0.00)$ & $0.16(0.00)$ & $0.37(0.00)$ & $0.24(0.00)$ & $0.13(0.00)$ & $-0.12(0.00)$ & $0.20(0.00)$ & $1.00(0.00)$ & $1.01(0.02)$ \\
\hline FS2 & 400 & $0.10(0.00)$ & $0.16(0.00)$ & $0.37(0.00)$ & $0.24(0.00)$ & $0.13(0.00)$ & $-0.13(0.00)$ & $0.20(0.00)$ & $0.91(0.04)$ & $0.45(0.34)$ \\
\hline FS3 & 400 & $0.10(0.00)$ & $0.16(0.00)$ & $0.37(0.00)$ & $0.24(0.00)$ & $0.13(0.00)$ & $-0.12(0.00)$ & $0.20(0.00)$ & $0.92(0.03)$ & $0.44(0.36)$ \\
\hline FS4 & 300 & $0.10(0.00)$ & $0.16(0.00)$ & $0.37(0.00)$ & $0.24(0.00)$ & $0.13(0.00)$ & $-0.12(0.00)$ & $0.20(0.00)$ & $1.01(0.01)$ & $0.99(0.02)$ \\
\hline FS5 & 300 & $0.10(0.00)$ & $0.16(0.00)$ & $0.37(0.00)$ & $0.24(0.00)$ & $0.13(0.00)$ & $-0.12(0.00)$ & $0.20(0.00)$ & $0.97(0.01)$ & $0.65(0.15)$ \\
\hline \multirow[t]{2}{*}{ FS6 } & 300 & $0.10(0.00)$ & $0.16(0.00)$ & $0.37(0.00)$ & $0.24(0.00)$ & $0.13(0.00)$ & $-0.12(0.00)$ & $0.20(0.00)$ & $0.98(0.00)$ & $0.67(0.13)$ \\
\hline & $\mathrm{N}$ & $\eta_{1}=0.25$ & $\eta_{2}=0.25$ & $\eta_{3}=0.25$ & $R_{1}^{2}=0.00$ & $R_{2}^{2}=0.00$ & $R_{3}^{2}=0.00$ & $p^{*}=0.30$ & $\beta=1.00$ & $\sigma_{z}=1.00$ \\
\hline \multicolumn{11}{|c|}{ Simulation: additive; estimation: additive } \\
\hline FS1 & 800 & $0.25(0.00)$ & $0.25(0.00)$ & $0.25(0.00)$ & $0.00(0.01)$ & $0.00(0.01)$ & $-0.00(0.01)$ & $0.30(0.00)$ & $0.95(0.1)$ & $0.99(0.10)$ \\
\hline \multicolumn{11}{|c|}{ Simulation: dominant; estimation: dominant } \\
\hline FS1 & 800 & $0.25(0.00)$ & $0.25(0.00)$ & $0.25(0.00)$ & $-0.00(0.02)$ & $-0.00(0.02)$ & $-0.00(0.02)$ & $0.30(0.00)$ & $0.96(0.1)$ & $1.00(0.08)$ \\
\hline \multicolumn{11}{|c|}{ Simulation: recessive; estimation: recessive } \\
\hline FS1 & 800 & $0.25(0.00)$ & $0.25(0.00)$ & $0.25(0.00)$ & $-0.00(0.01)$ & $-0.00(0.01)$ & $0.00(0.01)$ & $0.30(0.00)$ & $1.73(0.25)$ & $0.29(0.11)$ \\
\hline
\end{tabular}

A total of $\mathrm{n}=1200$ individuals were simulated. Standard deviations are given in parentheses. FS denotes family structure (see footnote of table 1); $\mathrm{n}$ is the number of families; baseline parameter is $\mu=-2.00$.

mates of $R$ were virtually unbiased, with somewhat larger standard errors under the null hypothesis $H_{0}: R=0$, around 0.01 for all settings. Estimates of $\beta$ and $\sigma_{z}$ were virtually unbiased for all settings in table 1 as well, but they had large standard errors. Both parameters, $\hat{\beta}$ and $\hat{\sigma}_{z}$ were somewhat underestimated for FS2 and FS3, for example, $\hat{\beta}=0.66(0.24)$ and $\hat{\sigma}_{z}=0.5(0.21)$ for FS2 for the dominant model with $R_{1}=0.0$. When we increased the sample size from 400 to 1000 families, the corresponding estimates were $\hat{\beta}=0.68(0.10)$ and $\hat{\sigma}_{z}=0.77(0.1)$ indicating slow convergence for these examples. For all settings presented in table 1 the estimates of $\beta$ however were never statistically significantly different from the true value $\beta=1$.

Similar patterns were seen for a two-locus model under the null hypothesis, summarized in the last part of table 2 . As assessed by the mean square error (MSE) of the estimates (data not shown), $\sigma_{z}$ was best estimated for FS1 and FS4, the family structures that included both parents. In summary, family structures that included parents yielded more precise estimates of $\sigma_{z}$. In both settings in table 1, the dominant model performed very well, reflecting a large number of affected individuals for each simulation.

On a computer with a single Pentium 4, $3 \mathrm{GHz}$ processor using Linux, computing the MLE for a single dataset comprised of 1000 families of type FS1, took 5.2 min for the random effect model and $4 \mathrm{~s}$ for the fixed effects model.

Tables 2 and 3 show results for simulations for haplotypes consisting of two observed loci for randomly sampled families and families ascertained on the basis of one or more affected member, respectively. The LD pattern of haplotypes with the disease allele was chosen such that two haplotypes $\left(\eta_{1}, \eta_{2}\right)$ were positively correlated with the disease locus $\left(R_{1}=0.24\right.$ and $\left.R=0.13\right)$. Again, in both tables haplotype frequencies were estimated accurately with very low standard errors. Interestingly, for random- 
Table 3. Mean estimates over 100 simulations for family ascertained on at least one affected offspring

\begin{tabular}{|c|c|c|c|c|c|c|c|c|c|c|}
\hline FS & $\mathrm{N}$ & $\eta_{1}=0.10$ & $\eta_{2}=0.16$ & $\eta_{3}=0.37$ & $R_{1}=0.24$ & $R_{2}=0.13$ & $R_{3}=-0.12$ & $p^{*}=0.20$ & $\beta=1.00$ & $\sigma_{z}=1.00$ \\
\hline \multicolumn{11}{|c|}{ Simulation: additive; estimation: additive } \\
\hline FS1a & 400 & $0.10(0.00)$ & $0.16(0.00)$ & $0.37(0.00)$ & $0.24(0.00)$ & $0.13(0.00)$ & $-0.12(0.00)$ & $0.20(0.00)$ & $1.02(0.02)$ & $0.99(0.01)$ \\
\hline FS2a & 400 & $0.10(0.00)$ & $0.16(0.00)$ & $0.37(0.00)$ & $0.24(0.00)$ & $0.13(0.00)$ & $-0.12(0.00)$ & $0.20(0.00)$ & $0.96(C$ & 18) \\
\hline FS3a & 400 & $0.10(0.00)$ & $0.16(0.00)$ & $0.37(0.00)$ & $0.24(0.00)$ & $0.13(0.00)$ & $-0.13(0.00)$ & $0.20(0.00)$ & $0.93(0.02)$ & $0.58(0.18)$ \\
\hline FS4a & 300 & $0.10(0.00)$ & $0.16(0.00)$ & $0.37(0.00)$ & $0.24(0.00)$ & $0.13(0.00)$ & $-0.12(0.00)$ & $0.20(0.00)$ & $1.01(0.01)$ & $0.99(0.01)$ \\
\hline FS5a & 300 & $0.10(0.00)$ & $0.16(0.00)$ & $0.37(0.00)$ & $0.24(0.00)$ & $0.13(0.00)$ & $-0.12(0.00)$ & $0.20(0.00)$ & $0.95(0.02)$ & $0.69(0.10)$ \\
\hline FS6a & 300 & $0.10(0.00)$ & $0.16(0.00)$ & $0.37(0.00)$ & $0.24(0.00)$ & $0.13(0.00)$ & $-0.12(0.00)$ & $0.20(0.00)$ & $0.96(0.01)$ & $0.70(0.10)$ \\
\hline \multicolumn{11}{|c|}{ Simulation: dominant; estimation: dominant } \\
\hline FS1a & 400 & $0.10(0.00)$ & $0.16(0.00)$ & $0.37(0.00)$ & $.24(0.00)$ & $0.13(0.00)$ & $-0.12(0.00)$ & $0.20(0.00)$ & $0(0.06)$ & 0.99( \\
\hline S2a & 400 & $10(0.00)$ & $6(0.00)$ & & $0)$ & 0.13 & $00)$ & 0.20 & .03) & $.22)$ \\
\hline S3a & 400 & 0 & 0.16 & & & & & & & .20) \\
\hline FS4a & 300 & 0 & 0.16 & & 0 & & & & & $0.01)$ \\
\hline FS5a & 00 & 0 & 0) & & 0) & 0.13 & 0) & 0.20( & & $0.67(C$ \\
\hline FS6a & 300 & $0.10(0.00)$ & $0.16(0.00)$ & $0.37(0.00)$ & $0.24(0.00)$ & $0.13(0.00)$ & $-0.13(0.00)$ & $0.20(0.00)$ & $0.85(0.07)$ & $0.70(0.11)$ \\
\hline \multicolumn{11}{|c|}{ recessive; estimation: recessive } \\
\hline FS1a & 400 & $0.10(0.00)$ & $0.16(0.00)$ & $.37(0.00)$ & $0.24(0.00)$ & $0.13(0.00)$ & $-0.12(0.00)$ & 0.20 & & $1.01(0.01)$ \\
\hline FS2a & 400 & $10(0.00)$ & $.16(0.00)$ & $0.37(0.00)$ & $0.24(0.00)$ & $0.13(0.00)$ & $-0.12(0.00)$ & $0.20(0.00)$ & $0.99(0.00)$ & $0.60(0.16)$ \\
\hline FS3a & 400 & $0.10(0.00)$ & $0.16(0.00)$ & $0.37(0.00)$ & $0.24(0.00)$ & $0.13(0.00)$ & $-0.12(0.00)$ & $0.20(0.00)$ & $0.99(0.00)$ & $0.60(0.17)$ \\
\hline FS4a & 300 & $0.10(0.00)$ & $0.16(0.00)$ & $0.37(0.00)$ & $0.24(0.00)$ & $0.13(0.00)$ & $-0.12(0.00)$ & $0.20(0.00)$ & $1.00(0.00)$ & $0.99(0.01)$ \\
\hline FS5a & 300 & $0.10(0.00)$ & $0.16(0.00)$ & $0.37(0.00)$ & $0.24(0.00)$ & $0.13(0.00)$ & $-0.12(0.00)$ & $0.20(0.00)$ & $0.99(0.00)$ & $0.70(0.10)$ \\
\hline FS6a & 300 & $0.10(0.00)$ & $0.16(0.00)$ & $0.37(0.00)$ & $0.24(0.00)$ & $0.13(0.00)$ & $-0.12(0.00)$ & $0.20(0.00)$ & $1.00(0.00)$ & $0.72(0.09)$ \\
\hline
\end{tabular}

A total of $\mathrm{n}=1200$ individuals were simulated. Standard deviations are given in parentheses. FS denotes family structure (see footnote of table 1); $\mathrm{n}$ is the number of families; baseline parameter is $\mu=-2.00$.

ly sampled families, $\beta$ was also estimated very accurately and with much lower standard errors than in the single locus setting in table 1 . The random effects variance $\sigma_{z}$ was again slightly underestimated for FS2 and FS3, for all modes of inheritance, for example $\hat{\sigma}_{z}=0.55(0.18)$ for the additive model. Similar to the single locus case, observations on parents are important for an accurate estimation of $\sigma_{z}$. Standard errors were lower for estimates based on the ascertained samples for all parameters.

We also assessed the impact of misspecification of the mode of inheritance on the parameter estimates. For computational efficiency we simulated the model without a random effect. We fitted the penetrance function with the additive model, that has been shown to be robust to misspecification of the mode of inheritance [17]. Estimates of the observed allele frequencies and $R$ under the null were unbiased. However, $\beta$ was slightly underestimated when data were simulated from the recessive and dominant model and $p^{*}$ was underestimated for the dominant and overestimated for the recessive model. Under the alternative, $R$ was overestimated for the dominant model and underestimated for the recessive model. However, there was no statistically significant bias in any of the parameter estimates, and thus we concluded that our model was reasonably robust under misspecification.

\section{Power of the Likelihood Ratio Test}

Table 4 gives the power of the LR test for no linkage of the disease locus with the observed loci as well as the power of the family based association test (FBAT) [8] applied to the same data. The null hypothesis in the FBAT is that there is no association or no linkage of observed markers with disease. While the FBAT does not require HW and is more robust, parental phenotype information is not used. For a binary trait the FBAT test statistic is $T=\sum_{i}\left\{X_{i}-E\left(X_{i}\right)\right\}[18]$, where $X_{i}$ is a genotype score for offspring $i$, similar to $X^{*}$ in our model. The expectation $E\left(X_{i}\right)$ is taken conditional on parental genotypes, and thus the marginal distribution of parental genotypes does not contribute to the statistic. As the FBAT therefore uses only offspring genotypes and phenotypes, this comparison demonstrates how the respective use of data influences the power of the tests. Both, FBAT and our LR test are locally optimal, if assumptions hold [8]. We used a standard implementation of FBAT and allowed it to optimize an intercept parameter involved in computing the $X_{i}$ [19]. We used 5000 simulations based on random samples of 400 nuclear families with one or two offspring. Under the null hypothesis of no LD, $H_{0}: R=0$, both tests maintained the level of $\alpha=0.05$, even under misspecification of the mode of inheritance for the LR test statistic (first section of table 4). Table 4 shows the 
Table 4. Power comparison of FBAT with the LR test based on 5000 replications

\begin{tabular}{|c|c|c|c|c|c|c|c|c|c|}
\hline$\eta_{1}$ & $R_{1}$ & $p^{*}$ & $\beta$ & Simulation & Estimation & LR & FBAT & $\mathrm{LR}^{1}$ & $\mathrm{FBAT}^{1}$ \\
\hline 0.3 & 0.0 & 0.3 & 2.0 & dominant & dominant & 0.053 & 0.047 & 0.058 & 0.060 \\
\hline 0.3 & 0.0 & 0.3 & 2.0 & dominant & recessive & 0.051 & 0.047 & 0.053 & 0.060 \\
\hline 0.3 & 0.0 & 0.3 & 2.0 & dominant & additive & 0.050 & 0.047 & 0.053 & 0.060 \\
\hline 0.3 & 0.0 & 0.3 & 2.0 & recessive & dominant & 0.067 & 0.051 & 0.076 & 0.054 \\
\hline 0.3 & 0.0 & 0.3 & 2.0 & recessive & recessive & 0.051 & 0.051 & 0.051 & 0.054 \\
\hline 0.3 & 0.0 & 0.3 & 2.0 & recessive & additive & 0.059 & 0.051 & 0.061 & 0.054 \\
\hline 0.3 & 0.0 & 0.3 & 2.0 & additive & dominant & 0.051 & 0.051 & 0.051 & 0.064 \\
\hline 0.3 & 0.0 & 0.3 & 2.0 & additive & recessive & 0.053 & 0.051 & 0.053 & 0.064 \\
\hline 0.3 & 0.0 & 0.3 & 2.0 & additive & additive & 0.052 & 0.051 & 0.049 & 0.059 \\
\hline 0.3 & 0.1 & 0.3 & 2.0 & dominant & dominant & 0.251 & 0.082 & 0.304 & 0.134 \\
\hline 0.3 & 0.1 & 0.3 & 2.0 & dominant & recessive & 0.234 & 0.082 & 0.229 & 0.129 \\
\hline 0.3 & 0.1 & 0.3 & 2.0 & dominant & additive & 0.240 & 0.080 & 0.274 & 0.129 \\
\hline 0.3 & 0.1 & 0.3 & 2.0 & recessive & dominant & 0.150 & 0.060 & 0.201 & 0.099 \\
\hline 0.3 & 0.1 & 0.3 & 2.0 & recessive & recessive & 0.122 & 0.060 & 0.198 & 0.099 \\
\hline 0.3 & 0.1 & 0.3 & 2.0 & recessive & additive & 0.136 & 0.060 & 0.203 & 0.099 \\
\hline 0.3 & 0.1 & 0.3 & 2.0 & additive & dominant & 0.401 & 0.118 & 0.510 & 0.221 \\
\hline 0.3 & 0.1 & 0.3 & 2.0 & additive & recessive & 0.376 & 0.118 & 0.446 & 0.221 \\
\hline 0.3 & 0.1 & 0.3 & 2.0 & additive & additive & 0.404 & 0.118 & 0.524 & 0.221 \\
\hline 0.3 & 0.2 & 0.3 & 2.0 & dominant & dominant & 0.719 & 0.176 & 0.812 & 0.373 \\
\hline 0.3 & 0.2 & 0.3 & 2.0 & dominant & recessive & 0.669 & 0.181 & 0.691 & 0.373 \\
\hline 0.3 & 0.2 & 0.3 & 2.0 & dominant & additive & 0.705 & 0.176 & 0.776 & 0.373 \\
\hline 0.3 & 0.2 & 0.3 & 2.0 & recessive & dominant & 0.302 & 0.092 & 0.520 & 0.239 \\
\hline 0.3 & 0.2 & 0.3 & 2.0 & recessive & recessive & 0.305 & 0.094 & 0.586 & 0.239 \\
\hline 0.3 & 0.2 & 0.3 & 2.0 & recessive & additive & 0.316 & 0.094 & 0.545 & 0.239 \\
\hline 0.3 & 0.2 & 0.3 & 2.0 & additive & dominant & 0.915 & 0.303 & 0.972 & 0.646 \\
\hline 0.3 & 0.2 & 0.3 & 2.0 & additive & recessive & 0.897 & 0.295 & 0.954 & 0.646 \\
\hline 0.3 & 0.2 & 0.3 & 2.0 & additive & additive & 0.923 & 0.295 & 0.976 & 0.646 \\
\hline
\end{tabular}

$\mathrm{N}=400$ random nuclear families were sampled, $\mu=-2.00$. Columns Simulation and Estimation list the penetrance model used for simulating and estimating, respectively. Columns LR/FBAT and LR $1 / \mathrm{FBAT}^{1}$ show the power for families with parents and 1 and 2 offspring, respectively.

power for the alternatives $R=0.1$ and $R=0.2$ and $\beta=2$. In all settings the power of the LR test was better than that of the FBAT, for example, for $R=0.2, \eta=p^{*}=0.3$, $\beta=2$ the LR test had power of $93 \%$ as compared to $30 \%$ for FBAT for the additive model. Interestingly, the power of the LR test was not strongly influenced by misspecification of the mode of inheritance. The influence of family structure reflects the fact, that parental genotype information is only partially used in FBAT.

\section{Data Example}

We analyzed data from a study on Alzheimer's disease [20], comprised of 131 families with a total of 656 individuals. For each person in the study 17 SNPs in the ApoE region were observed. Families who had genotypes inconsistent with Mendelian inheritance were excluded from the analysis. Such inconsistencies could arise from genotyping errors, wrong paternity/sample mix-up, but are most likely due to recombinations that formed new haplotypes in offspring. Recombinations are likely in this data set, since markers span over $1 \mathrm{Mb}$. Implications are discussed below. Families in this study were ascertained on the basis of having at least one affected offspring, leading to the likelihood:

$$
\begin{aligned}
& L(\theta)=\prod P\left(Y_{i 1}, \ldots, Y_{i n_{i}}, G_{i 1}, \ldots, G_{i i_{i}} \mid \sum_{j=3}^{n_{i}} Y_{i j} \geq 1\right) \\
& =\frac{\prod P\left(Y_{i 1}, \ldots, Y_{i n_{i}}, G_{i 1}, \ldots, G_{i n_{i}}\right)}{P\left(\sum_{j=3}^{n_{i}} Y_{i j} \geq 1\right)},
\end{aligned}
$$


Table 5. Data analysis for a single SNP analysis of the Alzheimer's data set

\begin{tabular}{|c|c|c|c|c|c|c|c|}
\hline SNP & Model & $\eta_{1}$ & $R$ & $p^{*}$ & $\beta$ & $l_{A}$ & $\mathrm{p}$ value \\
\hline SNP1006 & dominant & 0.64 & 0.82 & 0.72 & 1.25 & $-6.72 e+02$ & $7.90 \mathrm{e}-02$ \\
\hline SNP875 & dominant & 0.7 & -0.36 & 0.76 & 1.24 & $-6.67 e+02$ & $4.60 \mathrm{e}-01$ \\
\hline SNP886 & dominant & 0.72 & -0.43 & 0.68 & 1.28 & $-6.58 \mathrm{e}+02$ & $2.60 \mathrm{e}-01$ \\
\hline SNP988 & dominant & 0.59 & -0.85 & 0.49 & 1.41 & $-6.74 e+02$ & $8.7 e-05$ \\
\hline SNP888 & dominant & 0.28 & -0.64 & 0.57 & 1.34 & $-6.63 e+02$ & $9.2 \mathrm{e}-03$ \\
\hline SNP873 & dominant & 0.73 & 0.59 & 0.56 & 1.35 & $-6.55 e+02$ & $1.4 \mathrm{e}-02$ \\
\hline SNP952 & dominant & 0.71 & 0.49 & 0.68 & 1.27 & $-6.68 \mathrm{e}+02$ & $2.40 \mathrm{e}-001$ \\
\hline SNP528 & dominant & 0.38 & 0.89 & 0.43 & 1.47 & $-6.77 e+02$ & $6.5 e-07$ \\
\hline SNP992 & dominant & 0.29 & -0.87 & 0.76 & 1.24 & $-6.45 e+02$ & $6.90 \mathrm{e}-02$ \\
\hline SNP465 & dominant & 0.44 & -0.12 & 0.72 & 1.25 & $-7.07 e+02$ & $7.90 \mathrm{e}-01$ \\
\hline SNP457 & dominant & 0.52 & -0.25 & 0.64 & 1.30 & $-7.20 \mathrm{e}+02$ & $4.10 \mathrm{e}-01$ \\
\hline SNP471 & dominant & 0.55 & -0.26 & 0.63 & 1.30 & $-7.17 e+02$ & $4.30 \mathrm{e}-01$ \\
\hline SNP479 & dominant & 0.32 & 0.34 & 0.67 & 1.28 & $-6.19 e+02$ & $4.00 \mathrm{e}-01$ \\
\hline SNP497 & dominant & 0.56 & 0.09 & 0.64 & 1.29 & $-7.08 \mathrm{e}+02$ & $7.10 \mathrm{e}-01$ \\
\hline SNP491 & dominant & 0.99 & 0.13 & 0.67 & 1.28 & $-4.04 \mathrm{e}+02$ & $4.40 \mathrm{e}-01$ \\
\hline SNP459 & dominant & 0.52 & -0.59 & 0.73 & 1.25 & $-6.87 e+02$ & $3.7 \mathrm{e}-02$ \\
\hline SNP512 & dominant & 0.79 & -0.31 & 0.74 & 1.24 & $-6.09 e+02$ & $4.70 \mathrm{e}-01$ \\
\hline
\end{tabular}

$l_{\mathrm{A}}$ is the supremum of the likelihood under the alternative; the $\mathrm{p}$ value is based on a $\chi_{1}^{2}$ approximation of the LR statistics. Parameter estimates are given under the alternative. Significant $\mathrm{p}$ values are given in bold.

for analysis. $P\left(\sum_{j=3}^{n_{i}} Y_{i j} \geq 1\right)$ was computed by marginalizing over parental genotypes:

$$
\begin{aligned}
& P\left(\sum_{j=3}^{n_{i}} Y_{i j} \geq 1\right)=1-P\left(\sum_{j=3}^{n_{i}} Y_{i j}=0\right) \\
& =1-\sum_{g_{i 1}, g_{i 2}} P\left(g_{i 1}, g_{i 2}\right) \prod_{j=3}^{n_{i}} P\left(Y_{i j}=0 \mid G_{i j}-g_{i j}\right) P\left(G_{i j}=g_{i j} \mid g_{i M}, g_{i P}\right)
\end{aligned}
$$

Similar to the above ascertainment correction, missing phenotype and genotype information was handled by marginalizing over it. We analyzed the data set by conducting a haplotype analysis based on pairs of neighboring loci and also 17 single locus analyses. We fitted the likelihood using additive, dominant and recessive modes of inheritance. We used the logistic penetrance function with and without random effect. We let $\mu=-2$, corresponding to a disease risk of $13 \%$ in individuals without the disease allele, which is close to the prevalence of dementia in individuals aged $80-85$ years [21]. In a sensitivity analysis, we also used $\mu=-1$ and $\mu=-3$ and obtained comparable results. Implications of the choice of $\mu$ are discussed later. As estimates for $\sigma_{z}$ were always very close to zero, we show results for the penetrance function without a random effect. As the best fit for each of the SNPs/ haplotypes was provided by the dominant model, we report these results in tables 5 and 6 for the single locus and haplotype analysis. Confidence intervals (CIs) of the estimates were based on quantiles of the empirical bootstrap distribution function using 1000 bootstrap repetitions. On a $3 \mathrm{GHz}$ Pentium 4 processor the single locus analysis took $31 \mathrm{~s}$ and $34 \mathrm{~min}$ for the model without and with a random effect, respectively. The respective times for the two-locus analysis were $4.1 \mathrm{~min}$ and $5.1 \mathrm{~h}$.

The strongest haplotype association was observed for SNPs (SNP528, SNP992) with $R_{1}^{2}=0.9$. In the single locus analysis the largest $R$ was found for SNP528, $R=0.89$. These findings are not surprising, as SNP528 is located in the ApoE region that is believed to be causative for $\mathrm{Alz}$ heimer's disease [20]. Thus, the haplotype analysis of (SNP528, SNP992) showed the same correlation with the disease SNP as the single locus analysis. The estimates of $\beta$ for the dominant model varied between 1.24 and 1.46 for the single locus and the haplotype analysis. Estimates for $p^{*}$ varied between 0.43 and 0.82 in the single locus analysis. For the haplotype analysis, $\hat{p}^{*}$ was between 0.4 and 0.6 except for haplotypes at (SNP457, SNP471) and (SNP491, SNP459) for which $\hat{p}^{*}=1$. Parameter estimates for SNP528 were $\hat{\eta}_{1}=0.38(95 \%$ CI $0.32,0.44), \hat{R}^{2}=0.89$ (95\% CI 0.71, 0.99), $\hat{p}^{*}=0.43(95 \%$ CI $0.34,0.53)$ and $\hat{\beta}=$ 1.46 (95\% CI 1.29, 1.66). Parameter estimates for SNP528 with the random effect were $\left(\hat{\eta}_{1}, \hat{R}_{1}^{2}, \hat{p}^{2}, \hat{\sigma}_{a}\right)=(0.38,0.89$, $0.43,1.47,0.007)$. 
Table 6. Data analysis of the Alzheimers data set with the dominant model

\begin{tabular}{|c|c|c|c|c|c|c|c|c|c|c|}
\hline SNP & $\eta_{1}$ & $\eta_{2}$ & $\eta_{3}$ & $R_{1}$ & $R_{2}$ & $R_{3}$ & $p^{*}$ & $\beta$ & $l_{A}$ & $\mathrm{p}$ value \\
\hline SNP106, SNP875 & 0.36 & 0.34 & 0.30 & 0.00 & 0.00 & 0.00 & 0.40 & 1.468 & $-8.70 e+02$ & $8.10 \mathrm{e}-01$ \\
\hline SNP875, SNP886 & 0.66 & 0.06 & 0.05 & -0.33 & -0.15 & 0.16 & 0.66 & 1.291 & $-8.10 e+02$ & $8.10 \mathrm{e}-01$ \\
\hline SNP886, SNP988 & 0.38 & 0.23 & 0.32 & -0.56 & -0.25 & 0.77 & 0.44 & 1.462 & $-9.30 e+02$ & $9.10 \mathrm{e}-04$ \\
\hline SNP988, SNP888 & 0.25 & 0.03 & 0.31 & -0.38 & -0.16 & -0.38 & 0.47 & 1.434 & $-9.0 e+02$ & $6.80 \mathrm{e}-04$ \\
\hline SNP888, SNP873 & 0.10 & 0.69 & 0.21 & -0.23 & 0.49 & -0.38 & 0.72 & 1.264 & $-7.20 \mathrm{e}+02$ & $4.30 \mathrm{e}-08$ \\
\hline SNP873, SNP952 & 0.39 & 0.25 & 0.36 & 0.70 & -0.36 & -0.39 & 0.42 & 1.432 & $-8.90 e+02$ & $1.20 \mathrm{e}-03$ \\
\hline SNP952, SNP528 & 0.41 & 0.01 & 0.32 & 0.79 & -0.03 & -0.59 & 0.52 & 1.441 & $-9.0 \mathrm{e}+02$ & $6.80 \mathrm{e}-05$ \\
\hline SNP528, SNP992 & 0.01 & 0.27 & 0.39 & -0.08 & -0.3 & 0.90 & 0.44 & 1.392 & $-8.90 e+02$ & $1.60 \mathrm{e}-05$ \\
\hline SNP992, SNP465 & 0.16 & 0.29 & 0.12 & -0.62 & 0.07 & -0.22 & 0.72 & 1.269 & $-9.40 e+02$ & $3.90 \mathrm{e}-01$ \\
\hline SNP465, SNP457 & 0.27 & 0.24 & 0.18 & 0.31 & -0.14 & -0.54 & 0.58 & 1.334 & $-9.90 e+02$ & $3.20 \mathrm{e}-01$ \\
\hline SNP457, SNP471 & 0.50 & 0.04 & 0.00 & 0.01 & -0.04 & 0.00 & 1.00 & 1.198 & $-7.80 \mathrm{e}+02$ & $9.50 \mathrm{e}-01$ \\
\hline SNP471, SNP479 & 0.15 & 0.16 & 0.41 & 0.06 & 0.34 & -0.43 & 0.61 & 1.343 & $-9.0 e+02$ & $5.50 \mathrm{e}-01$ \\
\hline SNP479, SNP497 & 0.18 & 0.38 & 0.16 & 0.36 & -0.17 & -0.25 & 0.61 & 1.227 & $-8.70 e+02$ & $8.0 \mathrm{e}-01$ \\
\hline SNP497, SNP491 & 0.60 & 0.39 & 0.00 & 0.01 & 0.01 & 0.00 & 0.51 & 1.386 & $-7.30 e+02$ & $1.40 \mathrm{e}-01$ \\
\hline SNP491, SNP459 & 0.50 & 0.01 & 0.49 & 0.01 & -0.02 & -0.01 & 1.00 & 1.186 & $-7.10 \mathrm{e}+02$ & $9.90 e-01$ \\
\hline SNP459, SNP512 & 0.32 & 0.43 & 0.21 & -0.51 & 0.69 & -0.34 & 0.61 & 1.355 & $-8.90 e+02$ & $1.30 \mathrm{e}-01$ \\
\hline SNP952, SNP992 & 0.05 & 0.23 & 0.66 & 0.02 & -0.56 & 0.50 & 0.68 & 1.283 & & $5.7 \mathrm{e}-01$ \\
\hline
\end{tabular}

For haplotypes consisting of 2 SNPs. Refer to the main article for details. $l_{A}$ is the supremum of the likelihood under the alternative; the p value reflects the LR test (see table 5).

We also tested the global null hypothesis of no linkage disequilibrium in between any of the observed loci with the true disease associated locus using the likelihood ratio test statistic. In the single SNP analysis, the lowest $\mathrm{p}$ value was derived for $S N P 528, p=6.5 \times 10^{-7}$. For the adjacent SNPs SNP952 and SNP992, however, results were not significant with p values of 0.2 and 0.07 , respectively. The haplotype analysis showed the most significant finding for SNPs SNP888 and SNP873 with a p value of $4.3 \times$ $10^{-8}$. SNPs SNP888 and SNP873 were significant in the single locus analysis each with a $\mathrm{p}$ value of $<0.01$. This finding is remarkable since haplotype analysis of the non-adjacent SNPs SNP952 and SNP992 which flank SNP528 would not have shown a significant association $(p>0.2$, table 6).

\section{Discussion}

In this paper we proposed a latent disease locus model that allows one to estimate LD parameters between observed SNPs and the putative unobserved disease-associated SNP in the same genomic region, as well as a penetrance parameter associated with the disease genotype. Our simulations have shown that model parameters can be estimated reliably. Under the null hypothesis of no LD of the disease locus with observed loci, the parameters related to the latent disease locus, $p^{*}$ and $\beta$, had large standard errors. However, the detailed modeling of the underlying genetic mechanism leads to substantial power gains compared to more robust methods.

One limitation of our model is that violations of assumptions, for example, misspecification of the mode of inheritance, cause biases in parameter estimates. We also assume that the intercept parameter, $\mu$ in the penetrance function is known. In our data example, increasing $\mu$ lead to lower estimates of the genetic effects, $\beta$.

Our re-analysis of an Alzheimer's study emphasized the role of the ApoE gene in agreement with previous studies $[20,22]$. The putatively causal variants in the ApoE gene are composed of two SNPs forming three haplotypes: $\varepsilon 2, \varepsilon 3$ and $\varepsilon 4$ [22], with allele frequencies of 0.08 , 0.77 and 0.15 , respectively. All genotypes other than $\varepsilon 2 / \varepsilon 2$ are at risk with ORs of $\sim 3.3(\varepsilon 3 / \varepsilon 3), \sim 12.3(\varepsilon 3 / \varepsilon 4)$ and $\sim 63.3(\varepsilon 4 / \varepsilon 4)$. Since our model accommodates only a single disease allele, effects of several disease alleles are collapsed into a single penetrance parameter and allele frequency. Our estimate $\beta=1.4$ was thus somewhat higher than that of the most frequent at risk genotype $\varepsilon 3 / \varepsilon 3$ (59\%). The allele frequency of at risk genotypes agreed well with our estimate for the allele frequency of the disease allele. If the two ApoE SNPs had been observed di- 
rectly, an additive model for $\varepsilon 3$ could have modeled disease risk more efficiently.

In related work, Whittemore and $\mathrm{Tu}$ [11] considered the same basic penetrance model as ours, where the probability of disease was a function of an unobserved disease locus in LD with observed loci, resulting in $\delta_{i} \neq 0$ for some $i$. Similar to our model the logistic intercept $\mu$ was assumed to be known. The main focus in [11] was testing the null hypothesis of no association, formulated as $H_{0}$ : $\beta=0$. Under this null hypothesis, the corresponding score statistic does not depend on the disease locus, allowing for robust inference. In contrast, we require $\beta \neq 0$ and are able to quantify a genetic effect even if the true disease locus is in linkage equilibrium to observed markers. However, our simulations indicate that in that situation the content of information is low, and estimates of $\beta$ had large variances. The null hypothesis for our setting is $H_{0}: \delta_{i}=0$ for all $i$, that is we test for no linkage of the latent disease locus with the region under study. under this null hypothesis the phenotype distribution is independent of parameters $\eta_{i}$, and depends only on $p^{*}, \beta$ and a polygenic random effect $\left(\sigma_{z}\right)$. This setting is identical to segregation analysis.

A practical limitation of our model is that the number of parameters grows exponentially with the number of loci included in the analysis. In the analysis presented here we first considered each locus individually, and followed up with haplotypes comprised of two loci. Chen and Abecasis [23] proposed a similar two-stage approach for a model of genotype imputation in families on whole genome basis. As a general guideline, our model could be used with individual loci to reassess the association found in a prior screening stage. Models with and without random effect could be compared to assess which level of model complexity is needed. The next steps would then include sliding window analyses for two or three SNPs to pinpoint haplotypes that show associations with the disease allele. Finally, it can be worthwhile to analyze nonadjacent SNPs jointly in a haplotype analysis, if there is evidence for a combined effect, such as parameter estimates from previous stages, LD patterns or prior knowledge. To correct for multiple testing, methods based on $p$ values [24] may be most practial, as the use of permutation methods is difficult due to the computational burden of our model. A sliding window approach suggested for GWAs and case-control data [25] uses a permutation procedure that evaluates a min $\mathrm{p}$ statistic based on the score function. In principle, this approach is applicable and may warrant further investigation.

In conclusion, our model can be helpful in the fine mapping stage of an association study and to complement results from other analyses.

\section{Acknowledgements}

We used the Biowulf Linux cluster at the National Institutes of Health, Bethesda, MD (http://biowulf.nih.gov). We thank Eden Martin for providing the data set and the reviewers for helpful comments that lead to improvment of the paper. Part of this work was supported by DFG grant BO 1955/2-3.

\section{References}

1 Klein RJ, Zeiss C, Chew EY, Tsai JY, Sackler RS, Haynes C, Henning AK, San-Giovanni JP, Mane SM, Mayne ST, Bracken MB, Ferris FL, Ott J, Barnstable C, Hoh J: Complement factor $\mathrm{H}$ polymorphism in age-related macular degeneration. Science 2005;308:385-389.

$\checkmark 2$ Rioux JD, Xavier RJ, Taylor KD, Silverberg MS, Goyette P, Huett A, Green T, Kuballa P, Barmada MM, Datta LW, Shugart YY, Griffiths AM, Targan SR, Ippoliti AF, Bernard EJ, Mei L, Nicolae DL, Regueiro M, Schumm LP, Steinhart AH, Rotter JI, Duerr RH, Cho JH, Daly MJ, Brant SR: Genomewide association study identifies new susceptibility loci for Crohn disease and implicates autophagy in disease pathogenesis. Nat Genet 2007;39:596-604.

\3 Dina C, Meyre D, Gallina S, Durand E, Körner A, Jacobson P, Carlsson LMS, Kiess W, Vatin V, Lecoeur C, Delplanque J, Vaillant E, Pattou
F, Ruiz J, Weill J, Levy-Marchal C, Horber F, Potoczna N, Hercberg S, StunffCL, Bougnères P, Kovacs P, Marre M, Balkau B, Cauchi S, Chèvre JC, Froguel P: Variation in FTO contributes to childhood obesity and severe adult obesity. Nat Genet 2007;39:724-726.

4 Steinthorsdottir V, Thorleifsson G, Reynisdottir I, Benediktsson R, Jonsdottir T, Walters GB, Styrkarsdottir U, Gretarsdottir S, Emilsson V, Ghosh S, Baker A, Snorradottir S, Bjarnason H, Ng MCY, Hansen T, Bagger Y, Wilensky RL, Reilly MP, Adeyemo A, Chen Y, Zhou J, Gudnason V, Chen G, Huang H, Lashley K, Doumatey A, So WY, Ma RCY, Andersen G, Borch-Johnsen K, Jorgensen T, van Vliet-Ostaptchouk JV, Hofker $\mathrm{MH}$, Wijmenga C, Christiansen C, Rader DJ, Rotimi C, Gurney M, Chan JCN, Pedersen O, Sigurdsson G, Gulcher JR, Thorsteinsdottir U, Kong A, Stefansson K: A variant in CDKAL1 influences insulin response and risk of type 2 diabetes. Nat Genet 2007;39:770-775.

5 Yeager M, Orr N, Hayes RB, Jacobs KB, Kraft P, Wacholder S, Minichiello MJ, Fearnhead $\mathrm{P}$, Yu K, Chatterjee N, Wang Z, Welch R, Staats BJ, Calle EE, Feigelson HS, Thun MJ, Rodriguez C, Albanes D, Virtamo J, Weinstein S, Schumacher FR, Giovannucci E, Willett WC, Cancel-Tassin G, Cussenot O, Valeri A, Andriole GL, Gelmann EP, Tucker M, Gerhard DS, Fraumeni JF, Hoover R, Hunter DJ, Chanock SJ, Thomas G: Genomewide association study of prostate cancer identifies a second risk locus at $8 \mathrm{q} 24$. Nat Genet 2007;39:645-649.

6 Teng J, Risch N: The relative power of familybased and case-control designs for linkage disequilibrium studies of complex human diseases. ii. individual genotyping. Genome Res 1999;9:234-241. 
7 Spielman RS, McGinnis RE, Ewens WJ: Transmission test for linkage disequilibrium: the insulin gene region and insulin-dependent diabetes mellitus (IDDM). Am J Hum Genet 1993;52:506-516.

$\checkmark 8$ Rabinowitz D, Laird N: A unified approach to adjusting association tests for population admixture with arbitrary pedigree structure and arbitrary missing marker information. Hum Hered 2000;50:211-223.

$\checkmark 9$ Antoniou AC, Easton DF: Polygenic inheritance of breast cancer: Implications for design of association studies. Genet Epidemiol 2003;25:190-202.

10 Houlston RS, Peto J: The search for low-penetrance cancer susceptibility alleles. Oncogene 2004;23:6471-6476.

$\checkmark 11$ Whittemore AS, Tu IP: Detection of disease genes by use of family data. I. Likelihoodbased theory. Am J Hum Genet 2000;66: 1328-1340.

12 Whittemore AS: Estimating genetic association parameters from family data. Biometrika 2004;91:219-225.

13 Wang T, Weir B, Zeng ZB: A populationbased latent variable approach for association mapping of quantitative trait loci. Ann Hum Genet 2006;70:506-523.
14 International HapMap Consortium: A haplotype map of the human genome. Nature 2005;437:1299-1320.

15 Stram DO: Tag snp selection for association studies. Genet Epidemiol 2004;27:365-374.

16 Boehringer S: Estimation of association parameters in family based association studies. Ph.D. thesis, University Dortmund, Germany, Institute of Statistics, 2007.

17 Pfeiffer RM, Gail MH: Sample size calculations for population- and family-based casecontrol association studies on marker genotypes. Genet Epidemiol 2003;25:136-148.

18 Horvath S, Xu X, Laird NM: The family based association test method: strategies for studying general genotype-phenotype associations. Eur J Hum Genet 2001;9:301-306.

19 Laird NM, Horvath S, Xu X: Implementing a unified approach to family-based tests of association. Genet Epidemiol 2000;19(suppl 1): S36-S42.
20 Martin ER, Lai EH, Gilbert JR, Rogala AR, Afshari AJ, Riley J, Finch KL, Stevens JF, Livak KJ, Slotterbeck BD, Slifer SH, Warren LL, Conneally PM, Schmechel DE, Purvis I, Pericak-Vance MA, Roses AD, Vance JM: SNPing away at complex diseases: analysis of single-nucleotide polymorphisms around APOE in Alzheimer disease. Am J Hum Genet 2000;67:383-394.

21 Jorm AF, Korten AE, Henderson AS: The prevalence of dementia: a quantitative integration of the literature. Acta Psychiatr Scand 1987;76:465-479.

22 Corder EH, Saunders AM, Risch NJ, Strittmatter WJ, Schmechel DE, Gaskell PC, Rimmler JB, Locke PA, Conneally PM, Schmader KE: Protective effect of apolipoprotein e type 2 allele for late onset Alzheimer disease. Nat Genet 1994;7:180-184.

23 Chen WM, Abecasis GR: Family-based association tests for genomewide association scans. Am J Hum Genet 2007;81:913-926.

24 Hochberg Y, Benjamini Y: More powerful procedures for multiple significance testing. Stat Med 1990;9:811-818.

$>25$ Huang BE, Amos CI, Lin DY: Detecting haplotype effects in genomewide association studies. Genet Epidemiol 2007;31:803-812. 